\title{
Towards a Numerical Unitarity Approach for Two-loop Amplitudes in QCD
}

\author{
Harald Ita*† \\ Physikalisches Institut, Albert-Ludwigs-Universiät Freiburg D-79104 Freiburg, Germany \\ E-mail: harald.ita@physik.uni-freiburg.de
}

\begin{abstract}
The numerical unitarity approach has been important for obtaining reliable QCD predictions for the LHC. Here I discuss the extension of the approach beyond the leading quantum corrections for computing multi-loop amplitudes. The numerical unitarity approach requires a suitable parametrization of the loop integrands as a sum of terms that integrate to zero (surface terms) and master integrands. The construction and classification of suitable surface terms which match the propagators structures of Feynman amplitudes is the main technical advance discussed. A number of spin-offs for integral reduction and further formal questions are briefly reviewed.
\end{abstract}

Loops and Legs in Quantum Field Theory

24-29 April 2016

Leipzig, Germany

\footnotetext{
* Speaker.

${ }^{\dagger}$ I would like to thank the organisers for the opportunity to present this work.
} 


\section{Introduction}

During the next decade the experiments at the Large Hadron Collider (LHC) will deliver measurements of the S-matrix at the 5\% level. Theory predictions with comparable quality are challenging and time consuming to obtain. The long-term aim of the presented research is to provide automated theory tools for fixed-order computations in QCD in order to match the experimental precision. Here I discuss the new methods ref. [1] for the computations of two-loop scattering amplitudes required for next-to-next-to leading order (NNLO) predictions.

In general terms, any contribution that increases the breath, depth, precision and completeness of predictions is important. Breadth or variety in the final states facilitates indirect detection and characterization of new physics. Depth towards high-multiplicity final states allows to probe the kinematic dependence of interactions. Finally, the percent-level measurements of Standard Model (SM) cross sections requires precision predictions often including second-order quantum corrections. Although challenging to obtain phenomenological amplitudes have an increased value during the run-time of the LHC making multi-loop computations worthwhile in many ways.

During the last ten years a similar challenge was solved by the theory community. The automation of NLO computations in the QCD and electroweak coupling expansion $[3,2]$ lifted the understanding of the SM to the $10 \%$ level. Presently a limited number of NNLO predictions are available with two final-state objects [4]. Going beyond this multiplicity or increasing the number of scales requires advances of the state-of-the-art techniques. I follow one of the successful approaches for NLO computations, the unitarity approach $[6,7]$ and lay out an extension to multi-loop amplitudes. In particular, I focus on the variant of the unitarity methods well suited for numerical computations $[8,9,2]$.

\section{The role of unitarity and surface terms}

Amplitude computations relate Feynman amplitudes to a universal ansatz in terms of master integrals and their coefficients,

$$
\mathscr{A}^{2-\text { loop }}:=\int d^{D} \ell d^{D} \tilde{\ell} A_{\text {Feyn. }}^{2-\text { loop }}(\ell, \tilde{\ell})=\sum_{i \in \text { integral basis }} d_{i} \mathscr{I}_{i}, \quad \text { and } \quad \mathscr{I}_{i}:=\int d^{D} \ell d^{D} \tilde{\ell} \frac{m_{i}(\ell, \tilde{\ell})}{\rho^{0} \cdots \tilde{\rho}^{(\tilde{N}-1)}} .
$$

Here $\mathscr{I}_{i}$ denote master integrals defined by numerator insertions $m_{i}(\ell, \tilde{\ell})$ and the topologies fig. 1 . I focus the discussion on 2-loop amplitudes although the discussion is valid beyond this. The independent loop momenta are denoted by $\ell$ and $\tilde{\ell}$, external momenta by $p_{i}$ and inverse propagators are denoted by $\rho$-variables, e.g. $\rho^{i}=\left(\ell-q_{i}\right)^{2}-m_{i}^{2}$ with $q_{i}=\sum_{j} p_{j}$.

The role of unitarity methods is two-fold; on the one hand the equations (2.1) can be split up in to a fine-grained set using analytic properties of amplitudes (see e.g. [10]). On the other hand, the left-hand side of the master equation is simplified to tree-level on-shell expressions by cutting [11],

$$
\left.\mathscr{A}^{2-\text { loop }}\right|_{\operatorname{cut}_{(t, \mathscr{C})}}=\oint_{\mathscr{C}} \mu_{t}[d \alpha]_{t} \prod_{i \in \text { corners }} A_{i}^{\text {tree }}(\ell, \tilde{\ell})=\left.\sum_{j \in \text { cut topologies }} d_{j} \mathscr{I}_{j}\right|_{\operatorname{cut}_{(t, \mathscr{C})}}+\text { parent contributions .(2.2) }
$$

The variable $t$ labels the cut topologies (specified by the list of cut propagators), $\mu_{t}$ refers to the integration measure after cutting and $\alpha$ 's denote the remaining integration variables. Eqn. (2.2) holds 
up to 'parent contributions' which refers to integrals with additional (un-cut) propagators. When solving eqn. (2.2) iteratively in the number of cut propagators starting from cutting the maximal number of propagators, the parent contributions are already available when needed. Thus, eqn. (2.2) are linear equations for the coefficients $d_{i}$. As an improvement, one solves for the coefficients $d_{i}$ directly using integration contours $\mathscr{C}_{i}$ dual to the master integrals [12],

$$
\left.\mathscr{I}_{i}\right|_{\operatorname{cut}_{\left(t, \mathscr{C}_{j}\right)}}=\oint_{\mathscr{C}_{j}} \mu_{t}[d \alpha] m_{i}(\alpha)=\delta_{i}^{j}
$$

as manifest by inserting eqn. (2.3) into eqn. (2.2). The integration over multi-dimensional contours $\mathscr{C}$ are challenging which motivates the algebraic variant of the unitarity approach, described next.

Given a basis of integrands (as opposed to the integrands of master integrals) the algebraic relation,

$$
A_{\text {Feyn. }}^{2-\text { loop }}(\ell, \tilde{\ell})=\sum_{i \in \text { integrand basis }} \frac{\tilde{d}_{i} \tilde{m}_{i}(\ell, \tilde{\ell})}{\rho^{0} \cdots \tilde{\rho}^{(\tilde{N}-1)}}
$$

holds with yet to be determined coefficient functions $\tilde{d}_{i}$. Such integrand parametrizations have been developed in the recent years $[13,14,15]$.

The unitarity equations emerge, when solving the equations for the coefficients $\tilde{d}_{i}$. As above, one solves the eqn. (2.4) topology by topology using their propagator structure. One proceeds iteratively in the number of propagators starting from the maximal number of propagators. One considers each topology modulo expressions with fewer propagators $\left(\rho^{i} \sim 0\right)$. Geometrically this amounts to computing factorization limits which put loop-particles on-shell [9]. The Feynman amplitude factorizes into trees yielding,

$$
\lim _{(\ell, \tilde{\ell}) \rightarrow \text { cut }_{t} \text { value }} A_{\text {Feyn. }}^{2-\text { loop }}(\ell, \tilde{\ell})=\left.\prod_{i \in \text { corners }} A_{i}^{\text {tree }}(\ell, \tilde{\ell})\right|_{\text {cut }_{t}}=\left.\sum_{i \in \text { numerator basis }} \tilde{d}^{i} \tilde{m}_{i}(\ell, \tilde{\ell})\right|_{\text {cut }_{t}}+\text { parent contributions. }
$$

Technically the on-shell limits are identical to cutting but with the integration omitted. The linear equations for $\tilde{d}_{i}$ are algebraic and suited for numerical implementation. However, in order to obtain the loop amplitude the integrand basis has to be reduced to master integrals.

Here I discuss a more direct approach [1] inspired by one-loop computations [8, 9]. One decompose the integrand, i.e. the right-hand side of eqn. (2.4) directly into master integrands (associated to master integrals) and vanishing integrals (surface terms),

$$
A_{\text {Feyn. }}^{2-\text { loop }}(\ell, \tilde{\ell})=\sum_{i \in \text { master integrals }} \frac{d^{i} m_{i}(\ell, \tilde{\ell})}{\rho^{0} \cdots \tilde{\rho}^{(\tilde{N}-1)}}+\sum_{j \in \text { surface terms }} \frac{\hat{d}^{j} \widehat{m}_{j}(\ell, \tilde{\ell})}{\rho^{0} \cdots \tilde{\rho}^{(\tilde{N}-1)}},
$$

with the properties,

$$
\mathscr{S}_{j}=\int d^{D} \ell d^{D} \tilde{\ell} \frac{\widehat{m}_{j}(\ell, \tilde{\ell})}{\rho^{0} \cdots \tilde{\rho}^{(\tilde{N}-1)}}=0 .
$$

The algebraic equations (2.5) hold just as before and yield $d_{i}$ and $\hat{d}_{j}$. Thus one obtains directly the coefficients $d_{i}$ of the master integrals. The coefficient of the surface terms $\hat{d}_{j}$ are but required as auxiliary information when solving the algebraic equations (2.6). 


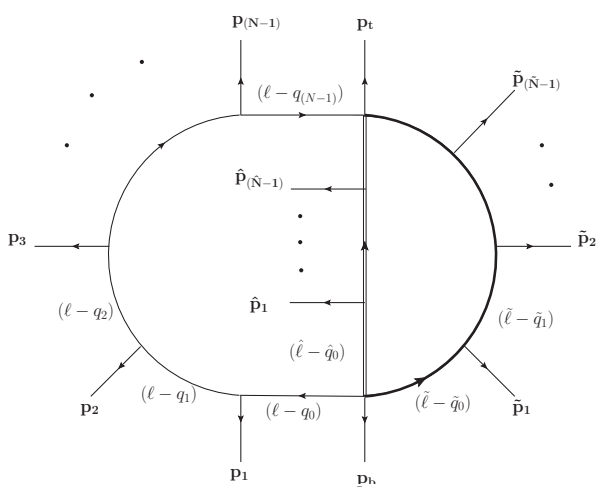

Figure 1: A generic two-loop integral topology is displayed with the naming conventions used in the main text. In order to reuse structures well known at one-loop level one interprets the two-loop topology as three strands. The strands carry loop momentum $\ell, \hat{\ell}$ and $\tilde{\ell}$, and have external momenta $p_{i}, \hat{p}_{i}, \tilde{p}_{i}, p_{t}$ and $p_{b}$ exiting.

An integrand representation in terms of surface terms and master integrands (2.6) has been suggested only recently [1]. Naively, integrands of IBP identities have all the properties needed, however, for unitarity cuts one requires very specific propagator powers. This obstruction is overcome in the construction of IBP-relations using a particular choice of 'IBP-generating' vectors [16]. I follow the methods developed in ref. [1] to explicitly construct and classify such IBP-generating vectors and obtain the surface terms topology by topology.

\section{Heuristic picture for loop integrals}

In order to clarify important mathematical structures, I start with a general discussion of loop integrals. A general coordinate change from the loop-momenta $\left(\ell^{\mu}\right.$ and $\left.\tilde{\ell}^{\mu}\right)$ to inverse propagators $\rho^{i}$ as integration variables [10] make the geometric structures of loop integrals manifest. Given the miss match in the number of propagators and loop-momentum components additional angular variables, $\alpha$ 's have to be introduced. If propagators are dependent on each other the integrals are reducible algebraically (using Gram determinants) and do not have to be considered.

The integration variables of the loop integrals are the inverse propagators and the $\alpha$-coordinates,

$$
\mathscr{I}_{i}=\int \frac{[d \rho]}{\rho^{0} \cdots \tilde{\rho}^{(\tilde{N}-1)}} \times \tilde{m}_{i} \mu_{t}[d \alpha]_{t}
$$

where $\tilde{m}:=\tilde{m}(\ell, \tilde{\ell})$ denotes a numerator insertion, $\mu_{t}$ is the non-trivial integration measure from the coordinate change. The expression $[d \rho]$ and $[d \alpha]_{t}$ denote the differentials of the integration variables; $\rho$ 's and $\alpha$ 's respectively. The integration makes a foliation of loop-momentum space manifest; for fixed propagator values one obtains an internal geometry parametrized by the $\alpha$ variables. The unitarity-cut phase space is one such internal slice.

It is instructive to consider first the integration over an internal space with the inverse propagators held fixed. One can now use the properties of the internal space to organize the computation. First of all, one can omit terms proportional to inverse propagators, since these will be captured by integrals with fewer propagators. For simplicity I focus on one-loop integrals next for which the 
$\alpha$-integration is performed over a sphere $\sum\left(\alpha^{a}\right)^{2}=C(\rho)[1,9,17]^{1}$. One can think of the function $\tilde{m}(0, \alpha)$ being expanded into spherical harmonics. Only the constant function gives a non-vanishing integral, while the higher harmonics integrate to zero. The latter can be interpreted as surface terms with the IBP-generating vectors being rotation generators in $\alpha$ space. Thus one is left with the non-vanishing integral with a constant numerator. IBP-generating vectors are tangent vectors of the internal space. In this way coordinates adapted to the propagator structures are a valuable tool for identifying reduction steps corresponding to canceling propagators and identifying vanishing integrals.

\section{Classifying surface terms}

Surface terms suited for perturbative computations are obtained from total derivatives

$$
\int d^{D} \ell d^{D} \tilde{\ell}\left[\partial_{\mu}\left(\frac{u^{\mu} \tilde{m}}{\rho^{0} \cdots \tilde{\rho}^{(\tilde{N}-1)}}\right)+\tilde{\partial}_{v}\left(\frac{\tilde{u}^{v} \tilde{m}}{\rho^{0} \cdots \tilde{\rho}^{(\tilde{N}-1)}}\right)\right]=0
$$

with polynomial vector fields $\left\{u^{\mu}, \tilde{u}^{v}\right\}$ and numerators $\tilde{m}:=\tilde{m}(\ell, \tilde{\ell})$. Propagators powers can be controlled by using 'IBP-generating vector-fields' fulfilling the equations [16],

$$
\left(u^{\mu} \partial_{\mu}+\tilde{u}^{v} \tilde{\partial}_{v}\right) \rho^{i}=f^{i}(\ell, \tilde{\ell}) \rho^{i},
$$

for all inverse propagators.

The relation (4.2) has an interesting on-shell interpretation. When specializing to the on-shell phase spaces with $\rho^{i}=0$, the vector fields $\left\{u^{\mu}, \tilde{u}^{v}\right\}$ turn into tangent vectors along the maximalcut phase spaces. It is often helpful to construct first the tangent vectors to the unitarity cut phase spaces and then take them off-shell in a second step.

In adapted coordinates the construction of IBP-generating vectors simplifies [1],

$$
\left(u^{a} \partial_{a}+u^{k} \partial_{k}\right) \rho^{i}=u^{i}=f^{i} \rho^{i} \rightarrow\left\{u^{a}, u^{i}\right\}=\left\{u^{a}, \rho^{i} f^{i}\right\},
$$

which follows from $\partial_{k} \rho^{i}=\delta_{k}^{i}$. The $i$ labels are not summed over in the above equation. I use the short-hand notation $\partial_{a}:=\partial_{\alpha^{a}}$ and $\partial_{k}:=\partial_{\rho^{k}}$. Additional constraints arise from requiring that $\left\{u^{a}, f^{i} \rho^{i}\right\}$ give polynomial vector fields in loop-momentum variables. I will deal with this constraint below in section 5 .

The physical meaning of the adapted coordinates suggests to introduce the following notation for IBP-generating vectors:

1. Horizontal vectors $\left\{u^{a}, u^{i}\right\}=\left\{u^{a}, 0\right\}$ inducing translations along slices of momentum space with fixed-propagator values.

2. Scaling vectors $\left\{u^{a}, u^{i}\right\}=\left\{f_{s} \alpha^{a}, \rho^{i} f^{i}\right\}$ inducing scaling transformations on the on-shell phase spaces. The corresponding IBP-relations depend on the dimension parameter D.

3. Vertical vectors $\left\{u^{a}, u^{i}\right\}=\left\{0, \rho^{i} f^{i}\right\}$ which vanish on the maximal cut, and point vertical to slices of fixed propagator values.

For the discussion of integrals with generic mass assignments the horizontal vectors are sufficient for constructing surface terms. I focus on this type below.

\footnotetext{
${ }^{1}$ Typically these spheres are part of the complex internal spaces which are tangent bundles of the real spheres $T S_{d}$.
} 


\section{Coordinates adapted to integral topology}

The general coordinate changes to coordinates adapted to integral topologies is obtained in two steps [1]. First for each strand in a multi-loop diagram a basis [5] of transverse vectors $n^{a}$, $\left(n^{a}, p_{j}\right)=0$ and dual vectors $v^{i}=\left(G^{-1}\right)^{i j} p_{j}$ is introduced using the Gram matrix $G_{i j}=\left(p_{i}, p_{j}\right)$. A distinct such basis is used per strand; two or three for two-loop diagrams. In a second step the basis change to inverse propagators is setup,

$$
\begin{gathered}
\ell(\rho, \alpha):=\sum_{i} r_{i} v^{i}+\sum_{a} \alpha^{a} n^{a}, \quad r_{i}:=-\frac{1}{2}\left(\left(\rho^{i}+m_{i}^{2}-q_{i}^{2}\right)-\left(\rho^{i-1}+m_{i-1}^{2}-q_{i-1}^{2}\right)\right), \\
q_{i}=\sum_{j=1}^{i} p_{j}, \quad c(\rho, \alpha):=\left(\ell^{2}-m_{0}^{2}\right)-\rho^{0}=0 .
\end{gathered}
$$

It is helpful not to eliminate an $\alpha$ variable using the constraint $c(\rho, \alpha)=0$. Instead one uses the redundant set of variables $\left\{\alpha^{a}, \rho^{i}\right\}$ and keeps the quadratic constraint $c(\alpha, \rho) \sim 0$ explicit. For multiple strands momentum conservation is imposed when strands join in a vertex. In summary, at one-loop level one has one constraint, while one introduces three constraints for planar two-loop integrals after solving for momentum conservation [1]. Below multiple constraints are labeled by an index; $c_{i}(\alpha, \rho)$.

Numerator insertions are polynomial functions in the loop momenta. It is an important property of the introduced coordinates, that numerators are again polynomials in $\alpha^{a}$ and $\rho^{i}$ variables,

$$
\left(t_{\mu_{1} \ldots v_{k}} \ell^{\mu_{1}} \ldots \tilde{\ell}^{v_{k}}\right) \longleftrightarrow \prod_{a, \tilde{a}, l, \tilde{l}}\left(\alpha^{a}\right)^{k_{a}}\left(\tilde{\alpha}^{\tilde{a}}\right)^{k_{\tilde{a}}} \times\left(\rho^{l}\right)^{k_{l}}\left(\tilde{\rho}^{\tilde{l}}\right)^{k_{\tilde{l}}}
$$

The variables $k_{i}$ denote non-negative integers. Polynomials that differ by a multiple of the constraints $c_{i}(\alpha, \rho)$ give identical loop-momentum functions as can be verified using eqn. (5.1).

Polynomial surface terms are obtained from polynomial vector fields in momentum space. Their defining property is that their directional derivatives map polynomials to polynomials. One obtains two types of restrictions; (1) when acting on the coordinate functions $\alpha^{a}$ and $\rho^{i}$ one finds that the components $\left\{f^{i} \rho^{i}, u^{a}\right\}$ are polynomials in $\alpha^{a}$ and $\rho^{i}$. (2) The equivalence of polynomials modulo the constraints is maintained only if the vector fields map the constraint ideal to itself,

$$
\left(\sum_{a} u^{a} \partial_{a}+\sum_{i} f^{i} \rho^{i} \partial_{i}\right) c_{j}(\alpha, \rho) \sim c_{k}(\alpha, \rho)
$$

Geometrically one reaches the same conclusion; the constraints $c_{i}(\alpha, \rho) \sim 0$ state, that the physical momentum space is a sub manifold $\left(c_{i}(\alpha, \rho)=0\right)$ in the $\{\alpha, \rho\}$-coordinate space. Vectors in loop-momentum space are naturally tangent vectors of this sub manifolds and thus give zero when acting on the constraints $c_{i}(\alpha, \rho)$ on the solution space of $c_{i}(\alpha, \rho)=0$ consistent with eqn. (5.4).

\section{Explicit surface terms}

The surface terms are obtained from total derivatives of IBP-generating vectors. The master integrals are obtained as numerators in the complement of the surface terms. This construction 
differs from the one at one-loop level $[8,9,17]$ which relied on tensor algebra and symmetries of one-loop integrals, but reproduces the results. All IBP-generating vectors are obtained from a set of primitive ones, which I list in the following. By inspection one can verify that the below vectors are horizontal IBP-generating vectors.

The one-loop IBP-generating vectors are given in standard notation by,

$$
u_{[k l]}=\left(\ell, n_{[k \mid}\right)\left(n_{\mid l]}, \partial\right) .
$$

The two-loop vectors are given by the following three types:

$$
\begin{aligned}
& \text { (a) } u_{[i j k]}=\left(\hat{\ell}, n_{[i \mid}\right)\left(\ell, n_{|j|}\right)\left(n_{\mid k]}, \partial\right), \quad\left(\text { b) } u_{[k l]}=\left(\ell, n_{[k \mid}\right)\left(n_{\mid l]}, \partial\right)+\left(\tilde{\ell}, \tilde{n}_{[k \mid}\right)\left(\tilde{n}_{\mid l]}, \tilde{\partial}\right),\right. \\
& \text { (c) } u_{[i j][k l]}=\left(\tilde{\ell}, \tilde{n}_{[k \mid}\right)\left(\tilde{n}_{\mid l]}, \hat{\ell}\right)\left(\ell, n_{[i \mid}\right)\left(n_{\mid j]}, \partial\right)-\left(\ell, n_{[i \mid}\right)\left(n_{\mid j]}, \hat{\ell}\right)\left(\tilde{\ell}, \tilde{n}_{[k \mid}\right)\left(\tilde{n}_{\mid l]}, \tilde{\partial}\right) .
\end{aligned}
$$

The vectors generate (a) rotations around an axis, (b) diagonal rotations and (c) crossed rotations, respectively. Tilde's indicate variables of a second sub-loop.

Multiplying the above vectors with generic tensors gives further IBP vectors. The surface terms are obtained by computing the divergence (4.1) with IBP vectors inserted. The numerators of the horizontal IBPvectors are given by

$$
\prod_{i, j}\left(n_{i}, \ell\right)^{k_{i}}\left(\tilde{n}_{j}, \tilde{\ell}\right)^{k_{j}} \times u \rightarrow \widehat{m}_{(u, \vec{k})}=\left(u^{\mu} \partial_{\mu}+\tilde{u}^{v} \tilde{\partial}_{v}\right)\left(\prod_{i, j}\left(n_{a}, \ell\right)^{k_{i}}\left(\tilde{n}_{\tilde{a}}, \tilde{\ell}\right)^{k_{j}}\right) .
$$

since the divergence of the horizontal vectors vanishes and they annihilate the propagators. The numerators $\widehat{m}_{u}(6.4)$ give the surface terms (4.1). This is true even if the powers of the propagators altered.

\section{Total derivatives and master-integrand count}

One requires a complete set of tensor insertions for a given integral topology. Systematic constructions of such a basis of tensor insertions can be found at one-loop level in in ref. [9] (see also [17]) and for multi-loop topologies in refs. [14, 15, 13].

For a given integral topology inserting an inverse propagator allows one to cancel a propagator and leads to a reduced topology. Thus one considers numerator tensors modulo inverse propagators $\left(\rho^{i} \sim 0\right)$. Comparing to (5.3) one identifies the independent numerator as the polynomials,

$$
\prod_{a, \tilde{a}}\left(\alpha^{a}\right)^{k_{a}}\left(\tilde{\alpha}^{\tilde{a}}\right)^{k_{\tilde{a}}}=\prod_{a, \tilde{a}}\left(n_{a}, \ell\right)^{k_{a}}\left(\tilde{n}_{\tilde{a}}, \tilde{\ell}\right)^{k_{\tilde{a}}}
$$

modulo the relations $c_{i} \sim 0$ and $\rho^{j} \sim 0$.

These equivalence relations amount to imposing the on-shell conditions, with all inverse propagators set to zero. Thus, linear independent numerator insertions are identified with independent functions on the maximal-cut phase spaces. In order to count master integrands one works on the unitarity cuts.

Cutting propagators amounts to replacing the propagators with delta-distributions,

$$
\int \frac{[d \rho]}{\rho^{0} \cdots \tilde{\rho}^{(\tilde{N}-1)}} \times\left.\tilde{m} \mu[d \alpha] \quad \stackrel{\text { cut }}{\longrightarrow} \int \tilde{m}(0, \alpha) \mu\right|_{\rho^{i}=0}[d \alpha]
$$


Terms with some of the cut propagators missing are omitted in the cutting prescription.

For the IBP-generating vectors $\left(u^{i}=f^{i} \rho^{i}\right)$ cutting total derivatives gives total derivatives on the unitarity cut surface,

$$
\int\left[\frac{\partial_{i}\left(f^{i} \mu \tilde{m}\right)}{\rho^{0} \cdots \lambda^{i} \cdots \tilde{\rho}^{(\tilde{N}-1)}}+\partial_{b}\left(\frac{u^{b} \mu \tilde{m}}{\rho^{0} \cdots \tilde{\rho}^{(\tilde{N}-1)}}\right)\right][d \rho, d \alpha] \stackrel{\text { cut }}{\longrightarrow} \quad \int \partial_{b}\left(u^{b} \mu \tilde{m}\right)[d \alpha] .
$$

The special property that the IBP-generating vector fields are tangent vectors of the maximal-cut phase spaces leads to this equation.

The number of master integrands is given by the number of independent tensor insertions modulo the number of surface terms. On-shell this amounts to the number of closed holomorphic forms modulo exact holomorphic forms (total derivatives), which is a topological index of the phase spaces. Thus a topological property, in fact the number of half-maximal cycles of the unitarity cut variety, counts master integrands.

\section{Summary and future directions}

I discussed new methods for the computation of multi-loop amplitudes using a numerical unitarity approach. The methods form a synthesis of established techniques to obtain integral relations (or surface terms) [16] and the unitarity approach.

I presented a number of results from ref.[1]: First of all, I setup the numerical unitarity approach for multi-loop amplitudes. I suggested a classification of integral relations and constructed a complete set of surface terms (6.4) for massive planar two-loop topologies. The surface terms are obtained from a new type of horizontal IBP-generating vectors. In addition, I pointed out the relation between the number of master integrands and topological properties of the unitarity-cut phase spaces. A central result is the geometric interpretation of IBP-generating vectors and convenient coordinates for dealing with multi-loop integrals. These methods appear to be a useful extension to the mathematical toolbox for loop computations.

The best combination of analytic and numerical approaches for multi-loop computations is not obvious in the moment, however, motivated by the one-loop successes of the unitarity method it seems worth while to follow a similar strategy for multi-loop computations. I am optimistic that the new methods discussed can contribute to precision predictions for the LHC experiments on the long run.

\section{Acknowledgments}

This work is supported by a Marie Skłodowska-Curie Action Career-Integration Grant PCIG12GA-2012-334228 of the European Union and by the Juniorprofessor Program of Ministry of Science, Research and the Arts of the state of Baden-Württemberg, Germany.

\section{References}

[1] H. Ita, [arXiv:1510.05626[hep-ph]].

[2] C. F. Berger, Z. Bern, L. J. Dixon, F. Febres Cordero, D. Forde, H. Ita, D. A. Kosower and D. Maitre, Phys. Rev. D 78 (2008) 036003. 
[3] K. Arnold et al., Comput. Phys. Commun. 180 (2009) 1661; S. Badger, B. Biedermann and P. Uwer, Comput. Phys. Commun. 182 (2011) 1674; V. Hirschi, R. Frederix, S. Frixione, M. V. Garzelli, F. Maltoni and R. Pittau, JHEP 1105 (2011) 044; G. Bevilacqua, M. Czakon, M. V. Garzelli, A. van Hameren, A. Kardos, C. G. Papadopoulos, R. Pittau and M. Worek, Comput. Phys. Commun. 184 (2013) 986; G. Cullen, N. Greiner, G. Heinrich, G. Luisoni, P. Mastrolia, G. Ossola, T. Reiter and F. Tramontano, Eur. Phys. J. C 72 (2012) 1889; G. Cullen et al., Eur. Phys. J. C 74 (2014) 8, 3001. F. Cascioli, P. Maierhofer and S. Pozzorini, Phys. Rev. Lett. 108 (2012) 111601; S. Actis, A. Denner, L. Hofer, A. Scharf and S. Uccirati, JHEP 1304 (2013) 037.

[4] S. Catani, L. Cieri, D. de Florian, G. Ferrera and M. Grazzini, Phys. Rev. Lett. 108 (2012) 072001; M. Czakon, P. Fiedler and A. Mitov, Phys. Rev. Lett. 115 (2015) 5, 052001; T. Gehrmann, M. Grazzini, S. Kallweit, P. MaierhÃúfer, A. von Manteuffel, S. Pozzorini, D. Rathlev and L. Tancredi, Phys. Rev. Lett. 113 (2014) 21, 212001; F. Cascioli et al., Phys. Lett. B 735 (2014) 311; M. Grazzini, S. Kallweit, D. Rathlev and A. Torre, Phys. Lett. B 731 (2014) 204; R. Boughezal, C. Focke, X. Liu and F. Petriello, Phys. Rev. Lett. 115 (2015) 6, 062002; A. Gehrmann-De Ridder, T. Gehrmann, E. W. N. Glover, A. Huss and T. A. Morgan, arXiv:1507.02850 [hep-ph]; X. Chen, T. Gehrmann, E. W. N. Glover and M. Jaquier, Phys. Lett. B 740 (2015) 147; R. Boughezal, C. Focke, W. Giele, X. Liu and F. Petriello, Phys. Lett. B 748 (2015) 5; R. Boughezal, F. Caola, K. Melnikov, F. Petriello and M. Schulze, Phys. Rev. Lett. 115 (2015) 8, 082003 ;

[5] W. L. van Neerven and J. A. M. Vermaseren, Phys. Lett. B 137 (1984) 241.

[6] Z. Bern, L. J. Dixon, D. C. Dunbar and D. A. Kosower, Nucl. Phys. B 425 (1994) 217; Z. Bern, L. J. Dixon, D. C. Dunbar and D. A. Kosower, Nucl. Phys. B 435 (1995) 59; R. Britto, F. Cachazo and B. Feng, Nucl. Phys. B 725 (2005) 275.

[7] A. Brandhuber, S. McNamara, B. J. Spence and G. Travaglini, JHEP 0510 (2005) 011 ;S. D. Badger, JHEP 0901 (2009) 049 .D. Forde, Phys. Rev. D 75 (2007) 125019.

[8] G. Ossola, C. G. Papadopoulos and R. Pittau, Nucl. Phys. B 763 (2007) 147 .

[9] R. K. Ellis, W. T. Giele and Z. Kunszt, JHEP 0803 (2008) 003; W. T. Giele, Z. Kunszt and K. Melnikov, JHEP 0804 (2008) 049; R. K. Ellis, W. T. Giele, Z. Kunszt and K. Melnikov, Nucl. Phys. B 822 (2009) 270.

[10] R. J. Eden, P. V. Landshoff, D. I. Olive, J. C. Polkinghorne, "The Analytic S-Matrix," Cambridge University Press (1966)

[11] R. E. Cutkosky, J. Math. Phys. 1, 429 (1960).

[12] D. A. Kosower and K. J. Larsen, Phys. Rev. D 85 (2012) 045017.

[13] P. Mastrolia and G. Ossola, JHEP 1111 (2011) 014 ;P. Mastrolia, E. Mirabella, G. Ossola and T. Peraro, Phys. Lett. B 718 (2012) 173 ;Phys. Rev. D 87 (2013) 8, 085026 ;Phys. Lett. B 727 (2013) 532 .

[14] S. Badger, H. Frellesvig and Y. Zhang, JHEP 1204 (2012) 055.

[15] Y. Zhang, JHEP 1209 (2012) 042.

[16] J. Gluza, K. Kajda and D. A. Kosower, Phys. Rev. D 83, 045012 (2011).

[17] H. Ita, J. Phys. A 44 (2011) 454005. 